\title{
Neutral polyfluoroalkyl substances in the atmosphere over the northern South China Sea ${ }^{\text {25 }}$
}

\author{
Senchao Lai ${ }^{\text {a, f, * }}$, Junwei Song a , Tianli Song a , Zhijiong Huang a, Yingyi Zhang a, \\ Yan Zhao a , Guicheng Liu ${ }^{a}$, Junyu Zheng ${ }^{a}$, Wenying Mi ${ }^{b}$, , Jianhui Tang ${ }^{\mathrm{d}}$, Shichun Zou ${ }^{\mathrm{e}}$, \\ Ralf Ebinghaus ${ }^{\mathrm{b}}$, Zhiyong Xie ${ }^{\mathrm{b}, * *}$ \\ ${ }^{a}$ Guangdong Provincial Key Laboratory of Atmospheric Environment and Pollution Control, School of Environment and Energy, South China University of \\ Technology, Guangzhou, China \\ ${ }^{\mathrm{b}}$ Helmholtz-Zentrum Geesthacht, Centre for Materials and Coastal Research, Institute of Coastal Research, Geesthacht, Germany \\ ${ }^{\mathrm{c}}$ MINJIE Analytical Laboratory, Geesthacht, Germany \\ ${ }^{\mathrm{d}}$ Key Laboratory of Coastal Zone Environmental Processes and Ecological Remediation, Yantai Institute of Coastal Zone Research, CAS, Yantai, China \\ e School of Marine Sciences, Sun Yat-Sen University, Guangzhou, China \\ ${ }^{\mathrm{f}}$ Department of Multiphase Chemistry, Max Planck Institute for Chemistry, Mainz, Germany
}

\section{A R T I C L E I N F O}

\section{Article history:}

Received 29 January 2016

Received in revised form

9 April 2016

Accepted 14 April 2016

Available online 23 April 2016

\section{Keywords:}

Neutral PFASs

Northern South China Sea

Long-range transport

Atmospheric decay

\begin{abstract}
A B S T R A C T
Neutral Polyfluoroalkyl substances (PFASs) in the atmosphere were measured during a cruise campaign over the northern South China Sea (SCS) from September to October 2013. Four groups of PFASs, i.e., fluorotelomer alcohols (FTOHs), fluorotelomer acrylates (FTAs), fluorooctane sulfonamides (FOSAs) and fluorooctane sulfonamidoethanols (FASEs), were detected in gas samples. FTOHs was the predominant PFAS group, accounting for 95.2-99.3\% of total PFASs ( PPFASs), while the other PFASs accounted for a small fraction of $\Sigma$ PFASs. The concentrations of $\Sigma$ PFASs ranged from 18.0 to $109.9 \mathrm{pg} \mathrm{m}^{-3}$ with an average of $54.5 \mathrm{pg} \mathrm{m}^{-3}$. The concentrations are comparable to those reported in other marine atmosphere. Higher concentrations of $\Sigma$ PFASs were observed in the continental-influenced samples than those in other samples, pointing to the substantial contribution of anthropogenic sources. Long-range transport is suggested to be a major pathway for introducing gaseous PFASs into the atmosphere over the northern SCS. In order to further understand the fate of gaseous PFASs during transport, the atmospheric decay of neutral PFASs under the influence of reaction with $\mathrm{OH}$ radicals and atmospheric physical processes were estimated. Concentrations of 8:2 FTOH, 6:2 FTOH and MeFBSE from selected source region to the atmosphere over the SCS after long-range transport were predicted and compared with the observed concentrations. It suggests that the reaction with $\mathrm{OH}$ radicals may play an important role in the atmospheric decay of PFAS during long-range transport, especially for shorted-lived species. Moreover, the influence of atmospheric physical processes on the decay of PFAS should be further considered.
\end{abstract}

(C) 2016 Elsevier Ltd. All rights reserved.

\section{Introduction}

Polyfluoroalkyl substances (PFASs) is a group of man-made organic compounds, involving a wide range of chemicals with different fluorocarbon chains $\left(\mathrm{CF}_{3}\left[\mathrm{CF}_{2}\right]_{\mathrm{n}-}\right)$ (Buck et al., 2011). Due to

\footnotetext{
* This paper has been recommended for acceptance by Hageman Kimberly Jill.

* Corresponding author. Guangdong Provincial Key Laboratory of Atmospheric Environment and Pollution Control, School of Environment and Energy, South China University of Technology, Guangzhou, China.

** Corresponding author.

E-mail addresses: sclai@scut.edu.cn (S. Lai), zhiyong.xie@hzg.de (Z. Xie).
}

the unique chemical stability and surface-active properties, PFASs have been widely used in inks, varnishes, waxes, fire-fighting foams, coating formulations, repellents for textiles, leather and paper products (Paul et al., 2009). These substances may be directly released into the environment during production, usage and disposal (Giesy and Kannan, 2002; Lindstrom et al., 2011; Paul et al., 2009). PFASs have raised more concerns for their persistence and bioaccumulation, as well as potential toxicity on the human and wildlife in environment (Giesy and Kannan, 2002; Houde et al., 2011; Prevedouros et al., 2006). Perfluorooctanoate (PFOA) and Perfluorooctane sulfonate (PFOS), two of the most widely known PFASs, have been detected in water (Ahrens, 2011; Zhang et al., 
2013; Zhao et al., 2012, 2014), air (Jahnke et al., 2007; Li et al., 2011; Liu et al., 2015; Shoeib et al., 2005), soil (Meng et al., 2013; Wang et al., 2013), wildlife (Houde et al., 2005; Lam et al., 2014; Schiavone et al., 2009) and human blood (Bao et al., 2011; Kubwabo et al., 2013; Shoeib et al., 2011).

Neutral PFASs in the atmosphere play an important role in the global cycle and distribution of PFASs. It was reported that the degradation of neutral precursors (e.g., fluorotelomer alcohols (FTOHs), fluorooctane sulfonamides (FOSAs) and fluorooctane sulfonamidoethanols (FASEs)) could contribute to the burden of PFOA and PFOS in the atmosphere (D'eon et al., 2006; Ellis et al., 2004; Martin et al., 2006). These precursors are usually volatile or semivolatile. Therefore, they are capable of escaping into the atmosphere during their manufacture and usage. They have been observed not only in urban areas (Ahrens et al., 2012; Dreyer and Ebinghaus, 2009; Li et al., 2011), but also in remote areas (Cai et al., 2012a; Del Vento et al., 2012; Shoeib et al., 2010; Wang et al., 2015; Xie et al., 2013, 2015). In smog chamber studies, the atmospheric lifetimes were estimated to be approximately 20 days for FTOHs and 20-50 days for FASAs due to their slow reaction with OH radicals (Ellis et al., 2003; Martin et al., 2006). The reaction with $\mathrm{OH}$ radicals is suggested as the most important degradation mechanism rather than other processes such as photolysis, wet and dry depositions, reactions with $\mathrm{NO}_{3}$ radicals, $\mathrm{Cl}$ atom and $\mathrm{O}_{3}$ etc. (Ellis et al., 2003). The neutral PFASs can react with $\mathrm{OH}$ radicals in the atmosphere during long-range transport, which has been suggested to be the major source of PFOA and PFOS in remote areas (D'eon et al., 2006; Ellis et al., 2004; Martin et al., 2006).

The South China Sea (SCS) is a marginal sea surrounded by the fast-developing regions with immense production of industrial products containing PFASs. As mentioned, the release of neutral PFASs could happen during production and usage. Under prevailing winds, air mass carrying neutral PFASs from the surrounding areas could be transported into the atmosphere of the SCS. Although the PFASs in surface water in the SCS has been reported in the previous studies (Cai et al., 2012b; Kwok et al., 2015; Yamashita et al., 2004), few research has been conducted to study the occurrence, sources and fates of atmospheric neutral PFASs over the SCS. Meanwhile, the loss mechanisms of gaseous PFASs have been suggested but only been investigated in laboratory studies. To our best knowledge, there is no study has been conducted to examine the decay of PFASs in the ambient atmosphere of the SCS till now.

In this study, we present the observation of neutral PFASs in gas phase during a cruise campaign in the northern SCS from September to October 2013. The objectives of this work were: (1) to determine the concentrations and composition of neutral PFASs in the atmosphere over the northern SCS; (2) to investigate the sources of neutral PFASs in the oceanic area; (3) to understand how neutral PFASs decay during long-range transport and the possible constraints. The results would help to improve the current understanding of the occurrence, sources, fates and the future perspectives of the neutral PFASs in coastal and oceanic areas.

\section{Experimental}

\subsection{Sampling information}

Ten sets of air samples including gas and particulate matter (PM) samples were collected on the research vessel Experiment III during the northern SCS campaign from September to October 2013 (See Fig. 1) (Lai et al., 2015; Zhao et al., 2016). An integrated high volume air sampler was placed in the upper deck to collect gas and PM samples simultaneously. The sampling unit contains an inlet equipped with a glass fiber filter (GFF, pore size $0.7 \mu \mathrm{m}$ ) to collect PM sample and a self-made column filled with XAD-2 resin was

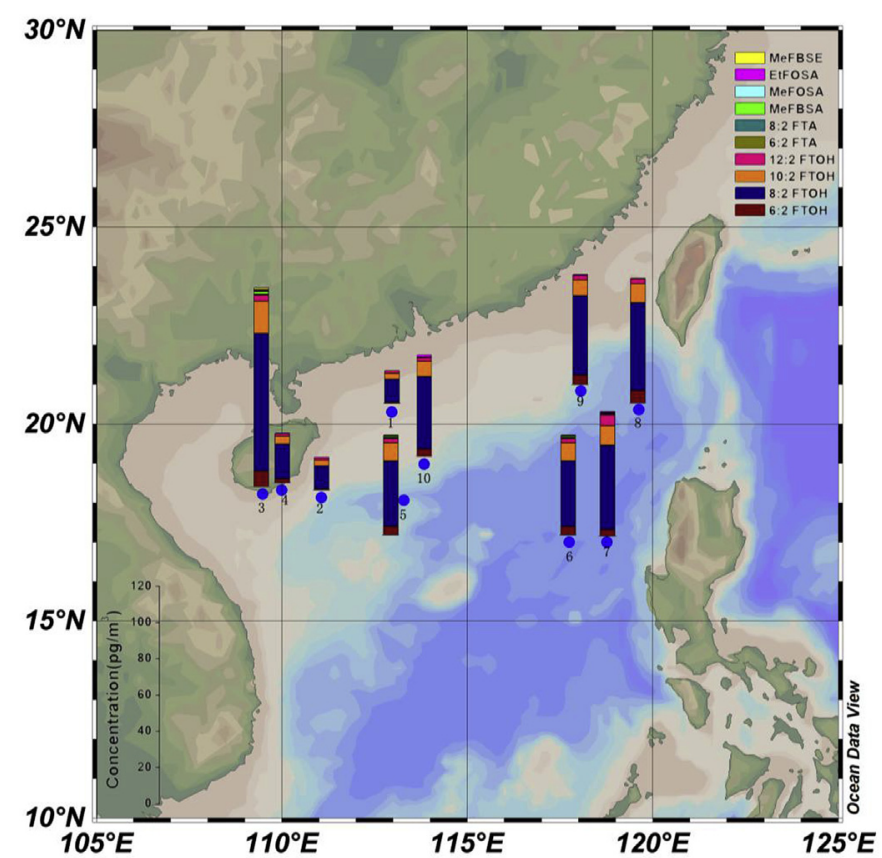

Fig. 1. Variations of neutral PFASs along the cruise in the atmosphere over the northern South China Sea.

followed to trap gaseous substances. The sampling volume of each air sample set ranged from $\sim 120$ to $\sim 450 \mathrm{~m}^{3}$. After collection, the samples were covered with prebaked aluminum foil bags and stored at $-20{ }^{\circ} \mathrm{C}$ until analysis. Detailed information of the air samples including date, latitude and longitude, sampling volume, air temperature, relative humidity and wind speed is listed in Table S1.

\subsection{Sample analysis}

The samples were spiked with $2.5 \mathrm{ng}$ of mass labeled internal standards prior to extraction. GFFs and columns were extracted using a MX extractor for $16 \mathrm{~h}$ using dichloromethane. Extracts were evaporated to $1-2 \mathrm{~mL}$ using hexane as a keeper and then passed through a column packed with $3 \mathrm{~g} \mathrm{Na}_{2} \mathrm{SO}_{4}$ to remove residual water. The extracts were further concentrated down to $150 \mu \mathrm{L}$ and spiked with $10 \mu \mathrm{L}$ of $100 \mathrm{pg} \mu \mathrm{L}^{-1}$ FTOH 9:1 as injection standard.

The method of sample analysis has been described elsewhere (Xie et al., 2013). Briefly, the analysis was performed using gas chromatography/mass spectrometry (GC/MS) in selective ion monitoring (SIM) mode using positive chemical ionization (PCI). The response factors were derived from the calibration curves (6points) made for response ratio between targets compounds $\left(0-0.25 n g \mu \mathrm{L}^{-1}\right)$ and corresponding surrogate $\left(0.05 \mathrm{ng} \mu \mathrm{L}^{-1}\right)$. The details of the analytes of FTOHs (6:2, 8:2, 10:2 and 12:2 FTOH), FASA (MeFBSA, MeFOSA and EtFOSA) and FASE (MeFBSE) are summarized in Table $\mathrm{S} 2$.

\subsection{Quality assurance/quality control ( $Q A / Q C)$}

Before sample collection, all resin columns were pre-cleaned with organic solvents (methanol, acetone and hexane/acetone in turn for $96 \mathrm{~h}$ ) and GFFs were baked at $550{ }^{\circ} \mathrm{C}$ for $12 \mathrm{~h}$ prior to their usage. The columns were covered with aluminum foil during sampling and stored in the aluminum foil bags after sampling to avoid the exposure to ultraviolet and visible light, which can cause the degradation of the target compounds. Contamination of ship 
exhaust was prevented by manually shutting off the sampling pump when the ship stopped or was under unfavourable wind conditions. Negligible influence of ship exhausts was suggested (Lai et al., 2015; Zhao et al., 2016). Field blank samples were taken during the campaign.

Breakthrough of the sampling methods has been checked previously at land base station using two columns connected in series. The upper column was spiked with $20 \mu \mathrm{L}$ of the internal standard mixture $\left(0.05 \mathrm{ng} \mu \mathrm{L}^{-1}\right)$. The recoveries of mass labeled $6: 2 \mathrm{FTOH}$, 8:2 FTOH, 10:2 FTOH, MeFOSA and EtFOSA were 91\%, 82\%, 85\%, 86\%, and $80 \%$ for $1500 \mathrm{~m}^{3}$ air sample, respectively. The values of individual PFASs ranged from 0.1 (6:2 FTOH, FTAs, MeFOSA, EtFOSA and MeFBSE) to $0.9 \mathrm{pg} \mathrm{m}^{-3}$ (8:2 FTOH) in the field blank samples. Method detection limits (MDLs) ranged from 0.1 to $2.1 \mathrm{pg} \mathrm{m}^{-3}$ (8:2 FTOH) in gas phase (details see Table S3).

\subsection{Models}

Seventy-two hour air mass back-trajectories at an altitude of $200 \mathrm{~m}$ were calculated along the sampling route using the Hybrid Single Particle Lagrangian Integrated Trajectory (HYSPLIT) model. The atmospheric transport of gaseous PFASs via advection, diffusion and deposition were simulated by using the Community Multiscale Air Quality (CMAQ) model. The detail model description is shown in supplement (Appendix A).

\section{Results and discussion}

\subsection{Neutral PFASs in the atmosphere}

Both gas and particle phase samples were collected during the cruise campaign in the northern SCS during September to October 2013. Ten PFASs species including 4 FTOHs (6:2, 8:2, 10:2 and 12:2 FTOH), 2 FTAs (6:2 and 8:2 FTA), 3 FASAs (MeFBSA, MeFOSA and EtFOSA) and a FASE (MeFBSE) were detected in gas samples but they could not be detected (below the MDLs) in particle phase. It shows that atmospheric PFASs exist dominantly in gas phase rather than in particle phase, which is consistent with the results from many previous studies (Stock et al., 2007; Xie et al., 2013, 2015). The concentrations of PFASs in each gas phase sample were presented in Fig. 1 (for details see Table S3). The concentration of total PFASs

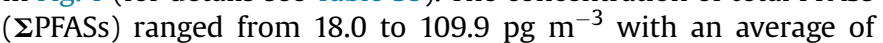
$54.5 \mathrm{pg} \mathrm{m}^{-3}$. Generally, the concentration of total FTOHs ( $\Sigma$ FTOHs) varied between 17.8 and $105.8 \mathrm{pg} \mathrm{m}^{-3}$ with an average of $53.0 \mathrm{pg} \mathrm{m}^{-3}$. Among the FTOHs, 8:2 FTOH was the predominant FTOH (12.5-75.8 $\mathrm{pg} \mathrm{m}^{-3}$, mean: $38.0 \mathrm{pg} \mathrm{m}^{-3}$ ), followed by $10: 2$ FTOH (3.3-17.8 pg m ${ }^{-3}$, mean: $8.1 \mathrm{pg} \mathrm{m}^{-3}$ ), 6:2 FTOH (0.7-8.6 pg m ${ }^{-3}$, mean: $4.4 \mathrm{pg} \mathrm{m}^{-3}$ ) and $12: 2$ FTOH (1.3-6.0 $\mathrm{pg} \mathrm{m}^{-3}$, mean: $2.5 \mathrm{pg} \mathrm{m}^{-3}$ ). The ratios of $8: 2 \mathrm{FTOH}$ to $10: 2$ FTOH to $6: 2$ FTOH can be used as an indicator to differentiate the emissions between local sources and atmospheric long-range transport (Li et al., 2011; Wang et al., 2015; Xie et al., 2015). In our study, the average ratio of $8: 2$ FTOH to $10: 2$ FTOH to $6: 2$ FTOH was 9.6: 2.1: 1.0. Similar ratios were observed in other regions, such as the Canadian Arctic (6.4: 2.1: 1.0) (Ahrens et al., 2011) and the North Sea (5.6: 2.5: 1.0) (Xie et al., 2013). However, lower ratios of $8: 2 \mathrm{FTOH}$ and 10:2 FTOH have been reported in the urban regions, e.g., Hamburg, Germany (1.1: 0.6: 1.0) (Dreyer and Ebinghaus, 2009) and Toronto, Canada (1.1: 0.2: 1.0) (Ahrens et al., 2012). The variation of the ratio could be affected by different source profile and also be attributed to the different removal rates of FTOHs in the atmosphere. The atmospheric residence times of FTOHs were estimated in field studies, with 80,70 and $50 \mathrm{~d}$ for $8: 2 \mathrm{FTOH}, 10: 2$ FTOH and 6:2 FTOH, respectively (Piekarz et al., 2007). The ratios of FTOHs observed in our study may point to the contribution of atmospheric long-range transport rather than local emissions.

The concentrations of FTAs were within the ranges of $<0.1-0.4 \mathrm{pg} \mathrm{m}^{-3}$ for 6:2 FTA and 8:2 FTA. FTAs have been widely used as the monomers in fluorotelomer production (Paul et al., 2009). The atmospheric lifetimes of FTAs are about several days, relatively shorter than those of FTOHs (Butt et al., 2009). The lower concentrations of FTAs than those of FTOHs in our dataset again suggest that the detected gaseous PFASs were related to long-range transport.

The concentrations of total FASA and FASEs (sum of MeFBSA, MeFOSA, EtFOSA and MeFBSE, $\Sigma$ FASA/SEs) ranged from 0.1 to $3.6 \mathrm{pg} \mathrm{m}^{-3}$ with an average of $1.2 \mathrm{pg} \mathrm{m}^{-3}$. MeFBSA and MeFBSE were two shorter chain-length chemicals, with the average concentrations of $1.2 \mathrm{pg} \mathrm{m}^{-3}$ and $0.8 \mathrm{pg} \mathrm{m}^{-3}$, respectively. Although production of perfluorooctane sulfonamide (FOSA) and perfluorooctane sulfonamidoethanol (FOSE) has been stopped and replaced with four carbon equivalent compounds since 2002 (Piekarz et al., 2007), concentrations of EtFOSA (0.1-1.3 $\mathrm{pg} \mathrm{m}^{-3}$ ) and MeFOSA $\left(<0.3-0.6 \mathrm{pg} \mathrm{m}^{-3}\right)$ were comparable to the concentration of MeFBSE. The levels of FOSA may be related to the products containing POSF-based chemicals in use (Paul et al., 2009).

The relative abundances of neutral PFASs in the gas samples were investigated (Fig. S1). FTOH was the most abundant PFAS group, accounting for $95.2-99.3 \%$ of $\Sigma$ PFASs, while short-lived compounds such as FTAs and FASAs/SEs in each gas samples accounted for less than $5 \%$ of $\Sigma$ PFASs. Among the FTOHs, the concentration of $8: 2$ FTOH contributed to $65.1-77.6 \%$ of $\Sigma$ PFASs, followed by $10: 2$ FTOH (4.4-18.5\%), 6:2 FTOH (3.8-11.4\%) and $12: 2$ FTOH (3.2-8.7\%). As suggested above, the predominance of FTOHs and lack of short-lived PFASs indicate that the influence of longrange transport is important for neutral PFASs in this region.

The concentrations of neutral PFASs were variable along the cruise track. The lowest $\Sigma$ PFASs concentration was observed in Sample A1 (18.0 $\mathrm{pg} \mathrm{m}^{-3}$ ), which was collected during cruising along the coastal area of southern China. The highest $\Sigma$ PFASs concentration was detected in Sample A3 $\left(109.9 \mathrm{pg} \mathrm{m}^{-3}\right)$ when the ship was on the way to Hainan Island. Sample A3 is suggested to be strongly influenced by the continental emissions based on the back-trajectory analysis (Fig. S2). Interestingly, Sample A4 and Sample A3 were collected in a close coastal area, when the ship was sailing to and leaving from Hainan Island, respectively. Lower concentration of $\Sigma$ PFASs was observed in Sample A4 (27.4 $\mathrm{pg} \mathrm{m}^{-3}$ ) than that in Sample A3. The $72 \mathrm{~h}$ back-trajectories show that Sample A4 was mainly influenced by the oceanic air masses. Oceanic emissions are not considered to be the substantial contributors for atmospheric PFASs since industrial production and usage of neutral PFASs are mainly located in human populated area. The rest samples were collected in open sea with the $\Sigma$ PFASs concentrations of $34.1-68.9 \mathrm{pg} \mathrm{m}^{-3}$. The variations of $\Sigma$ PFASs could be influenced by source regions, air mass transport and meteorological conditions (Li et al., 2011; Wang et al., 2015; Xie et al., 2013). In order to understand the influence of source regions, we classify the samples into two groups: continental (mainly from the Asian continent) and non-continental origins based on the air mass backtrajectories. Higher level of $\Sigma$ PFASs $\left(68.4 \pm 22.1 \mathrm{pg} \mathrm{m}^{-3}\right)$ was observed in continental samples (A3 and A6-A10) than those in non-continental samples (A1-A2 and A4-A5) $\left(33.6 \pm 15.7 \mathrm{pg} \mathrm{m}^{-3}\right)$. The results confirm that the Asian continent is an important source region for the long-range transport of neutral PFASs to the SCS. It is in accordance with the finding from our recent publication showing that atmospheric pollutants over the SCS are highly influenced by the continental outflow (Zhao et al., 2016). 


\subsection{Comparison with other reported gaseous PFASs}

An overview of $\Sigma$ FTOHs, $\Sigma$ FASAs/SEs and $\Sigma$ FTAs detected in different regions is shown in Fig. 2 (Ahrens et al., 2011, 2012; Cai et al., 2012a; Del Vento et al., 2012; Dreyer and Ebinghaus, 2009; Li et al., 2011; Shoeib et al., 2010; Wang et al., 2014, 2015; Xie et al., 2013, 2015). The concentrations of $\boldsymbol{\Sigma}$ FTOHs in this study were comparable with those observed in the marine atmosphere, e.g., the Atlantic to the Southern Ocean (Wang et al., 2015), the North Sea (Xie et al., 2013), the Japan Sea to the Arctic (Cai et al., 2012a) and the Northeast Atlantic Ocean (Shoeib et al., 2010), and lower than those in urban and coastal areas (Ahrens et al., 2012; Dreyer and Ebinghaus, 2009; Li et al., 2011; Wang et al., 2014). Relatively low concentrations of gaseous PFASs were observed in some remote sites, such as the Arctic Svalbard (Xie et al., 2015) and the western Atlantic Peninsula (Del Vento et al., 2012).

$\Sigma$ FASAs/SEs in our study was much lower than the values reported in the urban regions of the Asia-Pacific region (Fig. 2b) (Li et al., 2011), and also lower than those reported in other terrestrial areas (Del Vento et al., 2012; Wang et al., 2014). It should be noted that the observed concentrations in this study were even lower than those from the field measurements in other marine atmosphere, such as the North Sea (Xie et al., 2013) and the Japan Sea to Arctic Ocean (Cai et al., 2012a).

The concentrations of $\Sigma$ FTAs in this study was lower than those in other regions (Fig. 2c), especially in Japan (Li et al., 2011), the oceanic areas from Japan Sea to Arctic Ocean (Cai et al., 2012a) and the Atlantic to the Southern Ocean (Wang et al., 2015). The range of $\Sigma$ FTAs was similar with the values observed in rather remote areas, e.g., the Arctic Svalbard (Xie et al., 2015) and the western Atlantic Peninsula (Del Vento et al., 2012). Due to the short atmospheric lifetime (approximately 1 day), it implies that FTAs were collected during the ship cruising after long-range transport and the atmospheric conditions in the region could be favourable for atmospheric degradation.

\subsection{Correlations between neutral PFASs in gas phase}

Correlations between the individual PFASs in gas samples were shown in Table 1. Significantly positive correlations $(r>0.82$,
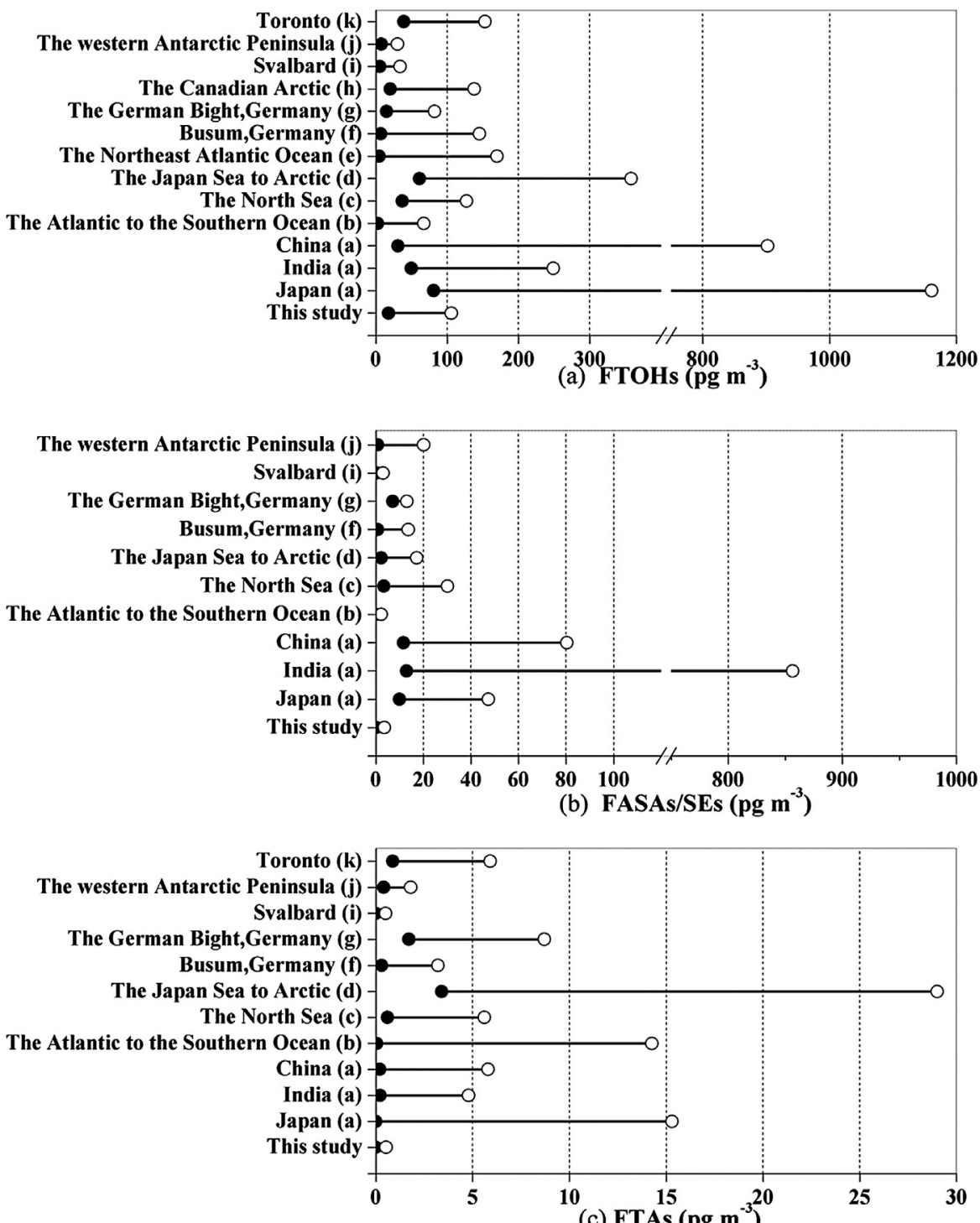

(c) FTAs (pg m $\left.{ }^{-3}\right)$

Fig. 2. Concentrations (minimum, maximum) of FTOHs, FASAs/SEs and FTAs in different regions ((a) Li et al., 2011, (b) Wang et al., 2015, (c) Xie et al., 2013, (d) Cai et al., 2012a, (e) Shoeib et al., 2010, (f) Wang et al., 2014, (g) Dreyer and Ebinghaus, 2009, (h) Ahrens et al., 2011, (i) Xie et al., 2015, (j) Del Vento et al., 2012, (k) Ahrens et al., 2012.). 
Table 1

Correlations ( $r$ ) between gaseous PFASs over the northern South China Sea.

\begin{tabular}{|c|c|c|c|c|c|c|c|c|c|}
\hline & $8: 2 \mathrm{FTOH}$ & 10:2 FTOH & 12:2 FTOH & $6: 2$ FTA & $8: 2$ FTA & MeFBSA & MeFOSA & EtFOSA & MeFBSE \\
\hline $6: 2 \mathrm{FTOH}$ & $0.902^{a}$ & $0.817^{a}$ & 0.305 & -0.035 & $0.864^{a}$ & 0.621 & $0.679^{\mathrm{b}}$ & 0.129 & 0.557 \\
\hline $8: 2 \mathrm{FTOH}$ & & $0.891^{\mathrm{a}}$ & 0.595 & 0.218 & $0.925^{a}$ & 0.575 & $0.735^{\mathrm{b}}$ & 0.233 & $0.694^{\mathrm{b}}$ \\
\hline 10:2 FTOH & & & 0.580 & 0.240 & $0.874^{a}$ & 0.301 & 0.580 & 0.234 & $0.673^{b}$ \\
\hline $12: 2$ FTOH & & & & $0.895^{\mathrm{a}}$ & 0.424 & -0.012 & 0.504 & 0.181 & 0.405 \\
\hline $6: 2$ FTA & & & & & 0.009 & -0.298 & 0.238 & 0.088 & 0.010 \\
\hline $8: 2$ FTA & & & & & & $0.680^{\mathrm{b}}$ & $0.782^{\mathrm{a}}$ & 0.087 & $0.860^{\mathrm{a}}$ \\
\hline MeFBSA & & & & & & & $0.758^{\mathrm{b}}$ & -0.201 & 0.567 \\
\hline MeFOSA & & & & & & & & 0.0690 & $0.672^{\mathrm{b}}$ \\
\hline EtFOSA & & & & & & & & & -0.095 \\
\hline
\end{tabular}

a Correlation is significant at the 0.01 level (2-tailed).

b Correlation is significant at the 0.05 level (2-tailed).

$\mathrm{p}<0.01$ ) were observed between 6:2 FTOH, 8:2 FTOH and 10:2 FTOH, suggesting that these FTOHs were derived from the common sources. The concentration of 12:2 FTOH shows weak correlation with other FTOHs, which may be related to other fluorotelomer processes. The concentration of 6:2 FTA is poorly correlated with 8:2 FTA, suggesting that they may originate from different source regions. Unexpectedly, 8:2 FTA has strong correlation with 6:2 FTOH, 8:2 FTOH and 10:2 FTOH ( $\mathrm{r}>0.86, \mathrm{p}<0.01)$, while 6:2 FTA significantly correlates with $12: 2$ FTOH $(r=0.89, \mathrm{p}<0.01)$. It has been proposed that significant correlations between PFASs may point to the common source regions and/or similar fates (Li et al., 2011; Piekarz et al., 2007). FTOHs can be used as the raw materials for FTAs manufacture during the fluorotelomer-based polymer production (Buck et al., 2011), e.g., 8:2 FTOH as a precursor in the production of 8:2 FTA (Prevedouros et al., 2006). Besides, FTOHs can lead to the formation of FTAs in the atmospheric degradation processes (Young and Mabury, 2010). The concentration of 8:2 FTA also exhibits significant correlation with those of MeFOSA $(r=0.78$, $\mathrm{p}<0.01)$ and MeFBSE $(\mathrm{r}=0.86, \mathrm{p}<0.01)$. Good correlations indicate that they have common source regions and/or with similar environmental fates.

\subsection{Estimation of neutral PFASs decay during transport}

The concentrations of organic compounds could be decreased during atmospheric dilution, wet and dry depositions, photolysis, and reactions with $\mathrm{OH}$ radicals, $\mathrm{NO}_{3}$ radicals, $\mathrm{Cl}$ atoms and $\mathrm{O}_{3}$ (Ellis et al., 2003; Yarwood et al., 2007). Smog chamber studies have suggested that the reaction with $\mathrm{OH}$ radicals would be the most important degradation pathway for neutral PFASs in the atmosphere (D'eon et al., 2006; Ellis et al., 2003; Martin et al., 2006). The atmospheric degradation processes of FTOHs have been reported as a source of FTAs (Young and Mabury, 2010). In addition, MeFBSE could be degraded by reacting with $\mathrm{OH}$ radicals to produce MeFBSA, which may contribute to the burden of poly-/perfluorinated pollutants in remote areas (D'eon et al., 2006). As discussed above, long-range transport was the major source of gaseous PFASs in the investigated region. Based on the air mass back-trajectory analysis, we further estimate the decay of PFASs along the air mass transport from several cities as the source areas to our cruise. The influence of $\mathrm{OH}$ reaction and atmospheric physical processes are investigated and discussed.

Firstly, the influence of the reaction with $\mathrm{OH}$ radicals is estimated. In the estimation, the reaction with $\mathrm{OH}$ radicals is assumed to be the only degradation pathway, based on Eqs. (1) and (2).

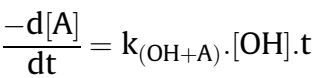

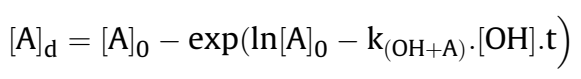

where $[A]_{0}$ is the concentration of a PFAS in a source city $\left(\mathrm{pg} \mathrm{m}^{-3}\right)$ which is obtained from the previous studies and is assumed to be in constant ranges; $[\mathrm{A}]_{\mathrm{d}}$ is the concentration of the same PFAS decay via $\mathrm{OH}$ radical reaction during transport $\left(\mathrm{pg} \mathrm{m}^{-3}\right) . \mathrm{K}_{(\mathrm{OH}+\mathrm{A})}$ is the reaction rate constant with $\mathrm{OH}$ radicals, and $\mathrm{t}$ is the transport time.

The source cities were selected based on the following criteria:

(1) The concentrations of gaseous PFASs are available in previous publications.

(2) The majority of the air masses back-trajectories of certain sample was originated from the lower atmosphere of the source city $(<500 \mathrm{~m})$.

(3) No other source region has been suggested during the air masses transport from the source city to the sampling site.

Based on the research of PFASs in the Asia-Pacific region (Li et al., 2011), we selected 3 cities (Guangzhou, Taichung and Hangzhou) as the source cities. The related samples are Sample A3, A6 and A7, respectively. The back-trajectories show that the air masses originated from these cities and mostly travelled over the oceanic areas when they were sampled. Three gaseous PFASs, i.e., 6:2 FTOH, 8:2 FTOH and MeFBSE, are chosen for our calculation due to the availability of the observation data and kinetic constants. We obtained the transport time from the source cities to the sampling sites according to the air mass back-trajectory analysis.

The kinetics of the reaction of $\mathrm{OH}$ radicals with FTOHs and MeFBSE were $\mathrm{K}_{(\mathrm{n}: 2 \mathrm{FTOH}+\mathrm{OH})}$ of $(1.07 \pm 0.22) \times 10^{-12} \mathrm{~cm}^{3} \mathrm{~mol}^{-1} \mathrm{~s}^{-1}$ and $\mathrm{K}_{(\mathrm{MeFBSE}+\mathrm{OH})}$ of $(5.8 \pm 0.8) \times 10^{-12} \mathrm{~cm}^{3} \mathrm{~mol}^{-1} \mathrm{~s}^{-1}$, respectively (D'eon et al., 2006; Ellis et al., 2003). The length of $F\left(\mathrm{CF}_{2} \mathrm{CF}_{2}\right)_{n-}$ group had no discernible impact on the reaction rate constants of FTOHs (Ellis et al., 2003). Referring to the previous observations of $\mathrm{OH}$ radicals in Southern China and in the western Pacific region (Hofzumahaus et al., 2009; Stone et al., 2012), a value of $1 \times 10^{6} \mathrm{~mol}$ $\mathrm{cm}^{-3}$ was adopted for the calculation in this study.

Besides the degradation reaction, the concentration decay of gaseous PFAS via atmospheric physical processes (e.g., advection, diffusion and deposition) should not be ignored during transport (Yarwood et al., 2007). Thus, we also estimated the influence of atmospheric physical processes on the concentration decay of PFASs by using CMAQ model. The influence of atmospheric dilution and dry deposition is mainly investigated in this simulation.

Based on the reaction decay with $\mathrm{OH}$ radicals and atmospheric physical processes decay by CMAQ the residual concentrations of PFASs in the sampling sites were predicted. The predicted residual concentrations of 3 gaseous PFASs (i.e., 6:2 FTOH, 8:2 FTOH and MeFBSE) after transport and observed concentrations in cruising samples are shown in Table 2. Except for the prediction of 6:2 FTOH 
Table 2

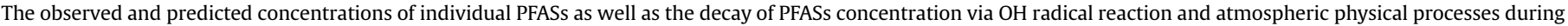
transport $\left(\mathrm{pg} \mathrm{m}^{-3}\right)$.

\begin{tabular}{|c|c|c|c|c|c|c|}
\hline \multirow[t]{2}{*}{ Cases } & \multirow{2}{*}{$\begin{array}{l}\text { Observed } \\
\text { concentrations }\end{array}$} & \multirow{2}{*}{$\begin{array}{l}\text { Source city } \\
\text { concentrations }^{\mathrm{a}}\end{array}$} & \multirow{2}{*}{$\begin{array}{l}\text { Transport time } \\
\text { (h) }\end{array}$} & \multicolumn{2}{|c|}{ Decay of PFASs concentration } & \multirow{2}{*}{$\begin{array}{l}\text { Predicted } \\
\text { concentrations }\end{array}$} \\
\hline & & & & $\begin{array}{l}\text { OH radical } \\
\text { reaction }^{\mathrm{b}}\end{array}$ & $\begin{array}{l}\text { Atmospheric physical } \\
\text { processes }^{c}\end{array}$ & \\
\hline Guangzhou $\rightarrow$ A3 & A3 & Guangzhou & & & & \\
\hline $6: 2 \mathrm{FTOH}$ & 8.6 & 81.6 & 34 & $10.0(12.3 \%)$ & $44.3(55.3 \%)$ & 27.3 \\
\hline $8: 2 \mathrm{FTOH}$ & 75.8 & 230.0 & 34 & $28.2(12.3 \%)$ & $153.0(66.5 \%)$ & 48.8 \\
\hline MeFBSE & 1.2 & 15.6 & 34 & $7.9(50.6 \%)$ & $8.3(53.5 \%)$ & -0.6 \\
\hline Taichung $\rightarrow$ A6 & A6 & Taichung & & & & \\
\hline $6: 2 \mathrm{FTOH}$ & 4.0 & 15.3 & 24 & $1.4(9.2 \%)$ & $12.1(79.2 \%)$ & 1.8 \\
\hline $8: 2 \mathrm{FTOH}$ & 35.6 & 709.0 & 24 & $62.6(8.8 \%)$ & $574.9(81.1 \%)$ & 71.5 \\
\hline MeFBSE & $<0.3$ & 16.2 & 24 & $6.4(39.5 \%)$ & $12.8(79.1 \%)$ & -3.0 \\
\hline Hangzhou $\rightarrow$ A7 & A7 & Hangzhou & & & & \\
\hline $6: 2$ FTOH & 3.6 & 20.3 & 36 & $2.6(12.8 \%)$ & $16.1(79.2 \%)$ & 1.6 \\
\hline $8: 2 \mathrm{FTOH}$ & 46.5 & 498.0 & 36 & $64.5(13.0 \%)$ & $387.2(77.8 \%)$ & 46.3 \\
\hline MeFBSE & 0.4 & 15.4 & 36 & $8.1(52.6 \%)$ & $11.7(75.9 \%)$ & -4.4 \\
\hline
\end{tabular}

a The concentrations of individual PFASs of source cities were from Li et al.

b A value of $1 \times 10^{6} \mathrm{~mol} \mathrm{~m}^{-3}$ was adopted for the calculation of $\mathrm{OH}$ radical reaction.

c The decay of PFASs concentration under atmospheric physical processes were based on the CMAQ model.

in Sample A3, there is generally good order-of-magnitude agreement between the predicted and observed concentrations of longer-lived PFASs (8:2 FTOH and 6:2 FTOH). Especially in Sample A7, the predicted residual concentration of 8:2 FTOH $\left(46.3 \mathrm{pg} \mathrm{m}^{-3}\right)$ was close to the observation $\left(46.5 \mathrm{pg} \mathrm{mg}^{-3}\right)$. It indicates that the prediction of the longer-lived PFASs decay during transport is acceptable. The reaction with $\mathrm{OH}$ radicals contributed only 8.8-13.0\% to the decay of longer-lived PFASs in all cases, which were lower than the contributions from atmospheric physical processes (55.3-81.1\%). It implies that the atmospheric physical processes may be the most important way for the decay of PFASs. MeFBSE is suggested to be a shorter-lived compound with an atmospheric lifetime of $\sim 2$ days (D'eon et al., 2006). Interestingly, all predicted MeFBSE concentrations were negative values, which may be due to the uncertainties of the prediction for shorted-lived PFASs. Compared with the predicted results, the observed concentrations of MeFBSE may be related to the background and/or other sources of MeFBSE in the marine atmosphere. Nevertheless, contributions of $\mathrm{OH}$ radical reaction to MeFBSE decay were 50.6\%, 39.5\% and 52.6\% in Sample A3, A6 and A7, respectively, which are close to the influence of atmospheric physical processes. It suggests that $\mathrm{OH}$ radical reaction could play a more important role in the decay of short-lived PFAS during transport. It should be noted that our prediction is quite crude and uncertainties could be caused by many aspects. First, the original PFAS concentrations in source city can only be derived from very limited observation data in East Asia. The reported concentrations could be influenced by many other factors such as seasons, synoptic conditions and local emission events. Second, observation data of $\mathrm{OH}$ radicals in ambient air are also limited and the reaction with $\mathrm{OH}$ radicals could be more complicated in the real atmosphere affected by the variation of $\mathrm{OH}$ radicals and other factors. Third, the simulation of atmospheric physical processes on the prediction was also limited. Therefore, observation of gaseous neutral PFASs is necessary to be conducted and the fates of PFASs in the atmosphere should be further investigated in the future.

\section{Conclusions}

Ten neutral PFASs were investigated in gas samples in a cruise campaign over the northern SCS during September-October 2013. The concentration levels of PFASs in our study were comparable to those measured in marine atmosphere in the previous studies. FTOHs were the predominant PFASs group, accounting for
95.2-99.3\% of 2 PFASs. The short-lived PFASs such as FTAs and FASEs showed lower concentrations than those of FTOHs, suggesting that atmospheric long-range transport was the major source of neutral PFASs. Air mass back-trajectory analysis indicates that the Asian continent was the most important source region of gaseous PFASs under the influence of long-range transport. To further understand the fate during transport, we calculated the decay of PFASs concentration via the $\mathrm{OH}$ radical reaction and atmospheric physical processes (i.e. atmospheric dilution and dry deposition) during transport, respectively. In most cases, the observed concentrations of longer-lived PFASs were in the same order-of-magnitude agreement with predicted ones. More important role of $\mathrm{OH}$ reaction is suggested in the decay of short-lived PFASs during transport. Due to the limited observation in the atmosphere of the SCS, more field measurements are necessary to monitor the occurrence of gaseous PFASs from continental area, to coastal and open sea areas. The investigation on the sink processes of PFASs in the marine environment is also of importance.

\section{Acknowledgments}

The authors thank the financial support of the National Natural Scientific Foundation of China (Grant No. 41105083, 41275130 and 41575115). Senchao Lai thanks the support of the Chinese Scholarship Council. The authors also thank Mr. Jinhu Peng and Mr. Zhanwen Zhang for their help on sample collection during the campaign.

\section{Appendix A. Supplementary data}

Supplementary data related to this article can be found at http:// dx.doi.org/10.1016/j.envpol.2016.04.047.

\section{References}

Ahrens, L., 2011. Polyfluoroalkyl compounds in the aquatic environment: a review of their occurrence and fate. J. Environ. Monit. 13, 20-31.

Ahrens, L., Harner, T., Shoeib, M., Lane, D.A., Murphy, J.G., 2012. Improved characterization of gas-particle partitioning for per- and polyfluoroalkyl substances in the atmosphere using annular diffusion denuder samplers. Environ. Sci. Technol. 46, 7199-7206.

Ahrens, L., Shoeib, M., Del Vento, S., Codling, G., Halsall, C., 2011. Polyfluoroalkyl compounds in the Canadian arctic atmosphere. Environ. Chem. 8, 399-406.

Bao, J., Liu, W., Liu, L., Jin, Y.H., Dai, J.Y., Ran, X.R., Zhang, Z.X., Tsuda, S., 2011. Perfluorinated compounds in the environment and the blood of residents living near fluorochemical plants in Fuxin, China. Environ. Sci. Technol. 45, 8075-8080. 
Buck, R.C., Franklin, J., Berger, U., Conder, J.M., Cousins, I.T., de Voogt, P., Jensen, A.A. Kannan, K., Mabury, S.A., van Leeuwen, S.P., 2011. Perfluoroalkyl and polyfluoroalkyl substances in the environment: terminology, classification, and origins. Integr. Environ. Assess. Manag. 7, 513-541.

Butt, C.M., Young, C.J., Mabury, S.A., Hurley, M.D., Wallington, T.J., 2009. Atmospheric chemistry of $4: 2$ fluorotelomer acrylate [C4F9CH2CH2OC(O)CH=CH2]: kinetics, mechanisms, and products of chlorine-atom- and oh-radical-initiated oxidation. J. Phys. Chem. A 113, 3155-3161.

Cai, M.H., Xie, Z.Y., Moller, A., Yin, Z.G., Huang, P., Cai, M.G., Yang, H.Z., Sturm, R. He, J.F., Ebinghaus, R., 2012a. Polyfluorinated compounds in the atmosphere along a cruise pathway from the Japan sea to the Arctic ocean. Chemosphere 87, 989-997.

Cai, M.H., Zhao, Z., Yang, H.Z., Yin, Z.G., Hong, Q.Q., Sturm, R., Ebinghaus, R., Ahrens, L., Cai, M.G., He, J.F., Xie, Z.Y., 2012b. Spatial distribution of per- and polyfluoroalkyl compounds in coastal waters from the east to south China sea. Environ. Pollut. 161, 162-169.

D'eon, J.C., Hurley, M.D., Wallington, T.J., Mabury, S.A., 2006. Atmospheric chemistry of $\mathrm{N}$-methyl perfluorobutane sulfonamidoethanol, C4F9SO2N(CH3) $\mathrm{CH} 2 \mathrm{CH} 2 \mathrm{OH}$ : kinetics and mechanism of reaction with $\mathrm{OH}$. Environ. Sci. Technol. 40, 1862-1868.

Del Vento, S., Halsall, C., Gioia, R., Jones, K., Dachs, J., 2012. Volatile per- and polyfluoroalkyl compounds in the remote atmosphere of the western Antarctic Peninsula: an indirect source of perfluoroalkyl acids to Antarctic waters? Atmos. Pollut. Res. 3, 450-455.

Dreyer, A., Ebinghaus, R., 2009. Polyfluorinated compounds in ambient air from ship- and land-based measurements in northern Germany. Atmos. Environ. 43, 1527-1535.

Ellis, D.A., Martin, J.W., De Silva, A.O., Mabury, S.A., Hurley, M.D., Andersen, M.P.S. Wallington, T.J., 2004. Degradation of fluorotelomer alcohols: a likely atmospheric source of perfluorinated carboxylic acids. Environ. Sci. Technol. 38, 3316-3321.

Ellis, D.A., Martin, J.W., Mabury, S.A., Hurley, M.D., Andersen, M.P.S., Wallington, T.J. 2003. Atmospheric lifetime of fluorotelomer alcohols. Environ. Sci. Technol. 37 3816-3820.

Giesy, J.P., Kannan, K., 2002. Perfluorochemical surfactants in the environment Environ. Sci. Technol. 36, 146-152.

Hofzumahaus, A., Rohrer, F., Lu, K.D., Bohn, B., Brauers, T., Chang C.C., Fuchs, H Holland, F., Kita, K., Kondo, Y., Li, X., Lou, S.R., Shao, M., Zeng, L.M., Wahner, A. Zhang, Y.H., 2009. Amplified trace gas removal in the troposphere. Science 324 1702-1704.

Houde, M., De Silva, A.O., Muir, D.C.G., Letcher, R.J., 2011. Monitoring of perfluorinated compounds in aquatic biota: an updated review PFCs in aquatic biota. Environ. Sci. Technol. 45, 7962-7973.

Houde, M., Wells, R.S., Fair, P.A., Bossart, G.D., Hohn, A.A., Rowles, T.K., Sweeney, J.C, Solomon, K.R., Muir, D.C.G., 2005. Polyfluoroalkyl compounds in free-ranging bottlenose dolphins (Tursiops truncatus) from the Gulf of Mexico and the Atlantic Ocean. Environ. Sci. Technol. 39, 6591-6598.

Jahnke, A., Berger, U., Ebinghaus, R., Temme, C., 2007. Latitudinal gradient of airborne polyfluorinated alkyl substances in the marine atmosphere between Germany and South Africa (53 degrees N-33 degrees S). Environ. Sci. Technol 41, 3055-3061.

Kubwabo, C., Kosarac, I., Lalonde, K., 2013. Determination of selected perfluorinated compounds and polyfluoroalkyl phosphate surfactants in human milk. Chemosphere 91, 771-777.

Kwok, K.Y., Wang, X.H., Ya, M.L., Li, Y.Y., Zhang, X.H., Yamashita, N., Lam, J.C.W. Lam, P.K.S., 2015. Occurrence and distribution of conventional and new classes of per- and polyfluoroalkyl substances (PFASs) in the South China Sea. J. Hazard. Mater. 285, 389-397.

Lai, S.C., Xie, Z.Y., Song, T.L., Tang, J.H., Zhang, Y.Y., Mi, W.Y., Peng, J.H., Zhao, Y., Zou, S.C., Ebinghaus, R., 2015. Occurrence and dry deposition of organophosphate esters in atmospheric particles over the northern South China Sea. Chemosphere 127, 195-200.

Lam, N.H., Cho, C.R., Lee, J.S., Soh, H.Y., Lee, B.C., Lee, J.A., Tatarozako, N., Sasaki, K., Saito, N., Iwabuchi, K., Kannan, K., Cho, H.S., 2014. Perfluorinated alkyl substances in water, sediment, plankton and fish from Korean rivers and lakes: a nationwide survey. Sci. Total Environ. 491, 154-162.

Li, J., Del Vento, S., Schuster, J., Zhang, G., Chakraborty, P., Kobara, Y., Jones, K.C., 2011 Perfluorinated compounds in the Asian atmosphere. Environ. Sci. Technol. 45, $7241-7248$.

Lindstrom, A.B., Strynar, M.J., Libelo, E.L., 2011. Polyfluorinated compounds: past, present, and future. Environ. Sci. Technol. 45, 7954-7961.

Liu, B.L., Zhang, H., Yao, D., Li, J.Y., Xie, L.W., Wang, X.X., Wang, Y.P., Liu, G.Q., Yang, B. 2015. Perfluorinated compounds (PFCs) in the atmosphere of Shenzhen, China: spatial distribution, sources and health risk assessment. Chemosphere 138, $511-518$.

Martin, J.W., Ellis, D.A., Mabury, S.A., Hurley, M.D., Wallington, T.J., 2006. Atmospheric chemistry of perfluoroalkanesulfonamides: kinetic and product studies of the $\mathrm{OH}$ radical and $\mathrm{Cl}$ atom initiated oxidation of $\mathrm{N}$-ethyl perfluorobutanesulfonamide, Environ. Sci. Technol, 40, 864-872.

Meng, J., Wang, T.Y., Wang, P., Giesy, J.P., Lu, Y.L., 2013. Perfluorinated compounds and organochlorine pesticides in soils around Huaihe River: a heavily contaminated watershed in central China. Environ. Sci. Pollut. Res. 20, 3965-3974.

Paul, A.G., Jones, K.C., Sweetman, A.J., 2009. A First global production, emission, and environmental inventory for perfluorooctane sulfonate. Environ. Sci. Technol. 43, 386-392.

Piekarz, A.M., Primbs, T., Field, J.A., Barofsky, D.F., Simonich, S., 2007. Semivolatile fluorinated organic compounds in Asian and western U.S air masses. Environ. Sci. Technol. 41, 8248-8255.

Prevedouros, K., Cousins, I.T., Buck, R.C., Korzeniowski, S.H., 2006. Sources, fate and transport of perfluorocarboxylates. Environ. Sci. Technol. 40, 32-44.

Schiavone, A., Corsolini, S., Kannan, K., Tao, L., Trivelpiece, W., Torres, D., Focardi, S., 2009. Perfluorinated contaminants in fur seal pups and penguin eggs from South Shetland, Antarctica. Sci. Total Environ. 407, 3899-3904.

Shoeib, M., Harner, T., Webster, G.M., Lee, S.C., 2011. Indoor sources of poly- and perfluorinated compounds (PFCS) in Vancouver, Canada: implications for human exposure. Environ. Sci. Technol. 45, 7999-8005.

Shoeib, M., Harner, T., Wilford, B.H., Jones, K.C., Zhu, J.P., 2005. Perfluorinated sulfonamides in indoor and outdoor air and indoor dust: occurrence, partitioning, and human exposure. Environ. Sci. Technol. 39, 6599-6606.

Shoeib, M., Vlahos, P., Hamer, T., Peters, A., Graustein, M., Narayan, J., 2010. Survey of polyfluorinated chemicals (PFCs) in the atmosphere over the northeast Atlantic Ocean. Atmos. Environ. 44, 2887-2893.

Stock, N.L., Furdui, V.I., Muir, D.C.G., Mabury, S.A., 2007. Perfluoroalkyl contaminants in the canadian arctic: evidence of atmospheric transport and local contamination. Environ. Sci. Technol. 41, 3529-3536.

Stone, D., Whalley, L.K., Heard, D.E., 2012. Tropospheric OH and HO2 radicals: field measurements and model comparisons. Chem. Soc. Rev. 41, 6348-6404.

Wang, P., Wang, T.Y., Giesy, J.P., Lu, Y.L., 2013. Perfluorinated compounds in soils from Liaodong Bay with concentrated fluorine industry parks in China. Chemosphere 91, 751-757.

Wang, Z., Xie, Z.Y., Mi, W.Y., Moller, A., Wolschke, H., Ebinghaus, R., 2015. Neutral poly/per-fluoroalkyl substances in air from the atlantic to the southern ocean and in Antarctic snow. Environ. Sci. Technol, 49, 7770-7775.

Wang, Z., Xie, Z.Y., Moller, A., Mi, W.Y., Wolschke, H., Ebinghaus, R., 2014. Atmospheric concentrations and gas/particle partitioning of neutral poly- and perfluoroalkyl substances in northern German coast. Atmos. Environ. 95, 207-213.

Xie, Z.Y., Wang, Z., Mi, W.Y., Moller, A., Wolschke, H., Ebinghaus, R., 2015. Neutral poly-/perfluoroalkyl substances in air and snow from the Arctic. Sci. Rep. 5, 8912.

Xie, Z.Y., Zhao, Z., Moller, A., Wolschke, H., Ahrens, L., Sturm, R., Ebinghaus, R., 2013. Neutral poly- and perfluoroalkyl substances in air and seawater of the North Sea. Environ. Sci. Pollut. Res. 20, 7988-8000.

Yamashita, N., Kannan, K., Taniyasu, S., Horii, Y., Okazawa, T., Petrick, G., Gamo, T., 2004. Analysis of perfluorinated acids at parts-per-quadrillion levels in seawater using liquid chromatography-tandem mass spectrometry. Environ. Sci. Technol. 38, 5522-5528.

Yarwood, G., Kemball-Cook, S., Keinath, M., Waterland, R.L., Korzeniowski, S.H., Buck, R.C., Russell, M.H., Washburn, S.T., 2007. High-resolution atmospheric modeling of fluorotelomer alcohols and perfluorocarboxylic acids in the north American troposphere. Environ. Sci. Technol. 41, 5756-5762.

Young, C.J., Mabury, S.A., 2010. Atmospheric perfluorinated acid precursors: chemistry, occurrence, and impacts. Rev. Environ. Contam. Toxicol. 208, 1-109.

Zhang, Y.Y., Lai, S.C., Zhao, Z., Liu, F.B., Chen, H.W., Zou, S.C., Xie, Z.Y., Ebinghaus, R. 2013. Spatial distribution of perfluoroalkyl acids in the Pearl River of southern China. Chemosphere 93, 1519-1525.

Zhao, Y., Zhang, Y., Fu, P., Ho, S.S.H., Ho, K.F., Liu, F., Zou, S., Wang, S., Lai, S., 2016. Non-polar organic compounds in marine aerosols over the northern south China Sea: influence of continental outflow. Chemosphere 153, 332-339.

Zhao, Y.G., Wan, H.T., Wong, M.H., Wong, C.K.C., 2014. Partitioning behavior of perfluorinated compounds between sediment and biota in the Pearl River Delta of South China. Mar. Pollut. Bull. 83, 148-154.

Zhao, Z., Xie, Z.Y., Moller, A., Sturm, R., Tang, J.H., Zhang, G., Ebinghaus, R., 2012. Distribution and long-range transport of polyfluoroalkyl substances in the Arctic, Atlantic Ocean and Antarctic coast. Environ. Pollut. 170, 71-77. 